\title{
Determination of crack surface orientation in carbon fibre reinforced polymers by measuring electromagnetic emission
}

\author{
S. O. Gade ${ }^{1}$. B. B. Alaca ${ }^{1}$. M. G. R. Sause ${ }^{2}$
}

Received: date / Accepted: date

\begin{abstract}
Electromagnetic emission (EME) generated by fracture of carbon fibre reinforced polymers (CFRP) is studied. The fracture is induced to cross-ply CFRP by mechanical loading in a three-point bending configuration. An EME acquisition set-up operating on the principle of capacitive coupling is used to measure the low frequency ( $\mathrm{kHz}-\mathrm{MHz}$ range) electric field whose generation is attributed to the charge redistribution accompanying the fracture processes. Multiple, differently oriented EME sensors, for the simultaneous EME measurement with different source-sensor orientations, were applied to account for the directionality of the EME sources and their generated electric fields. A method to deduce the crack orientation based on the emitted EME field's directionality is proposed. A comparison between the angles of the EME sources obtained by this method and the actual crack surface orientations as determined by computed tomography is made.
\end{abstract}

Keywords electromagnetic emission - fracture . carbon fibre reinforced plastics · source orientation

\section{Introduction}

The emission of particles and electromagnetic fields during and after fracture of solid materials is summarized

\footnotetext{
${ }^{1}$ Institute of Physics, Experimentalphysik II, University of Augsburg

Universitätsstrasse 1, 86159 Augsburg, Germany

${ }^{2}$ Institute for Materials Resource Management, Mechanical Engineering, University of Augsburg

Universitätsstrasse 1, 86159 Augsburg, Germany

${ }^{1}$ E-mail: sebastian.gade@physik.uni-augsburg.de

${ }^{2}$ E-mail: markus.sause@mrm.uni-augsburg.de
}

under the term fracto-emission. In this context a restriction to only the electromagnetic fields emitted by fracture is commonly referred to as electromagnetic emission (EME). This term also includes electromagnetic fields generated by other mechanical processes like plastic deformation or friction. EME effects over a wide range of frequencies were observed for a large variety of materials, e.g. rocks, minerals and concrete $[1,2,3,4$, $5]$, glass $[6,7,8]$, metals [9], polymers $[10,11]$, ice $[12,13]$ or polymer-matrix composites $[14,15,16]$ and concrete composites [17]. The mechanisms of EME generation have been subject to investigation for many years and multiple theories for the sources of EME in different materials and for different failure modes have been proposed. In general, the temporal characteristics of EME signals generated by fracture are correlated to a change in charge distribution and it's dynamics, both dictated by the fracture processes. Therefore, fracture induced EME signals contain valuable information about their sources. While acoustic emission (AE) signals, which are measured as transient displacements at the material's surface, are altered along their propagation path, EME signals can be detected almost undisturbed by the materials properties and geometry. This may prove particularly beneficial in complex micro structured materials like composites. These materials are designed to exhibit unique physical properties but also exhibit complex failure behaviour. Carbon fibre reinforced polymers (CFRP) are especially attractive for certain applications, e.g. in light weight engineering. Nevertheless, these materials are still subject to ongoing development and improvement in terms of theoretical descriptions, physical and mechanical properties of composites and their individual components as well as production processes. For the purpose of improving the performance of structures and the material itself, a detailed under- 
standing of the complex failure modes is essential. Although there has been some research concerning EME generated by fracture of fibre-reinforced polymers and its components in the past $[18,19,20,21]$, the full potential of the EME analysis has yet to be explored. A potentially useful application is offered by the characteristic field distribution of the fracture-generated EME signals. Assuming asymmetrical electrification of the crack surfaces, the cracks can be approximated as surface dipoles with corresponding electric dipole fields. EME with a distinct directionality has already been reported for other materials [11,22,23,24]. In this study we present results of EME measurements conducted during fracture tests of CFRP specimens with different ply stacking sequences. We particularly focus on the directionality of the emitted EME signals. Furthermore, we discuss the possibility to determine the orientation of inter-fibre cracks such as matrix cracking and delamination in cross-ply CFRP specimens.

\section{Experimental}

For the detection of EME generated by fracture of CFRP a three point bending test was conducted to induce a variety of failure mechanisms in cross-ply CFRP specimens. Figure 1 shows a schematic of the applied test set-up. The three point flexure test set-up was built in accordance with the geometry specified in DIN EN ISO 14125 [25]. For the test specimens, ply stacking sequences of $\left[0,90_{3}\right]_{\text {sym }},\left[0_{2}, 90_{3}\right]_{\text {sym }},\left[0,90_{2}, 0,90\right]_{\text {sym }}$ and $\left[0_{2}, 90,0,90\right]_{\text {sym }}$ were chosen. The specimens dimensions are $95 \mathrm{~mm}$ x $15 \mathrm{~mm}$ x 2.0-2.2 mm. All tested CFRP specimens were fabricated from unidirectional Sigrafil CE1250-230-39 carbon/epoxy prepreg laminate following the manufacturers recommendations for curing (90 minutes of curing in heat press with vacuum bagging, at $130^{\circ} \mathrm{C}$ and $0.7 \mathrm{MPa}$ ). All specimens were conditioned and tested at standard climate conditions $\left(23^{\circ} \mathrm{C} \pm 2{ }^{\circ} \mathrm{C}\right.$, $50 \% \pm 10 \%$ relative humidity), in accordance to DIN EN ISO 291 [26].

The mechanical test fixtures are usually made from steel, i.e. highly conductive material. As presented in [21], conductors placed near an EME source significantly influence it's field distribution and strength. For this reason, the test fixtures presented in the following sections were manufactured from non-conductive materials, mainly PMMA. Supports and load noses were made from PVC. A tube of pultruded glass-fibre-reinforced plastic (GFRP) is used to introduce the mechanical load.

Using test fixtures made from plastics naturally increases the compliance of the set-ups, which can be accounted for in the data reduction. Furthermore, the

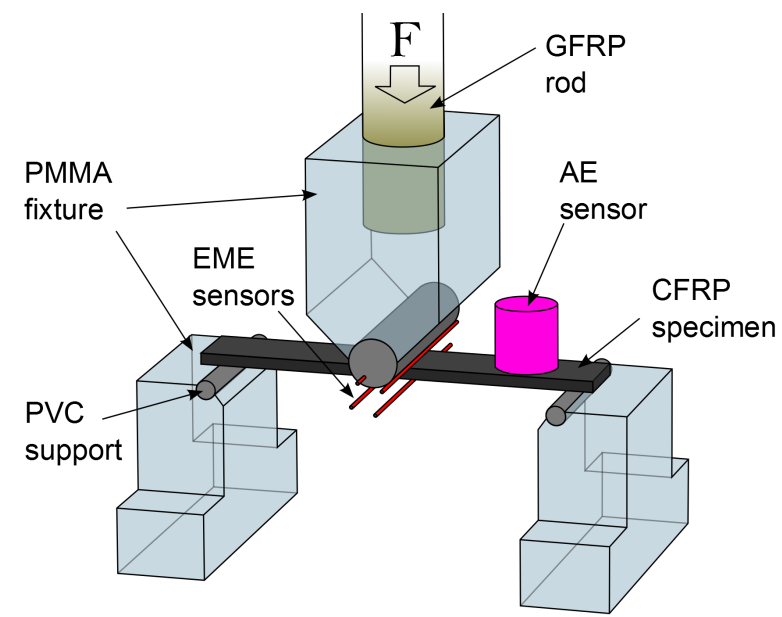

Fig. 1 Schematic of flexure test set-up with detail of EME sensor set-up.

compliance was measured to be constant within the load range considered in this investigation and no plastic deformation of the test fixtures was observed.

The AE signal detection was carried out using a high sensitivity wideband differential (WD) AE sensor (Physical Acoustics) attached to the surface of the specimen. The AE signals were amplified by $40 \mathrm{~dB}$ by a 2/4/6 pre-amplifier without internal bandpass filter and recorded by a PCI-2 acquisition card (Mistras corporation, software: AEwin). As acquisition parameters a threshold of $30 \mathrm{~dB}_{A E}$ and a sampling rate of $10 \mathrm{MS} / \mathrm{s}$ proved adequate.

For the EME detection the used mechanical testing set-ups were electrically shielded by a grounded metallic shielding enclosure. Two pairs of copper wires were used to measure the occurring electromagnetic fields via capacitive coupling $[27,28]$. The sensor pairs are arranged perpendicular to each other to account for the emitted electric field's directionality. The EME signals detected by these sensors were amplified in two stages. A first preamplifier is directly connected to the EME sensors, i.e. is located within the shielding enclosure. For this internal preamplifier a junction field effect transistor (n-channel JFET 2SK932-22) in a common source circuit with a $10 \mathrm{M} \Omega$ input resistor was used. The voltage signal then is further amplified by a low frequency amplifier (UBBV-NF35, Aaronia AG). The total amplification was set to $40 \mathrm{~dB}$. The EME signals are also recorded by the PCI-2 acquisition card. As EME signal acquisition parameters a threshold of $40 \mathrm{~dB}_{A E}$ and a sampling rate $10 \mathrm{MS} / \mathrm{s}$ were used. The band width of the acquisition system is limited by the $1 \mathrm{kHz}-3$ $\mathrm{MHz}$ band bass filter of the acquisition board. Between these cut-off frequencies the amplification of the EME acquisition set-up was evaluated to be constant. 
The AE and EME signals were chosen to be acquired in a synchronized mode. Thus, a triplet of $\mathrm{AE}$ and EME signals was recorded for every event, even when the much weaker EME signals did not exceed their threshold value. Furthermore, the AE acquisition also serves as a kind of filter, i.e. EME signals detected without corresponding $\mathrm{AE}$ signals were considered to not have originated from crack initiation or propagation and were therefore not considered for the analysis.

The mechanical load was applied displacement controlled by an universal testing machine (Zwick ZT 5.0). The cross head velocities was $1 \mathrm{~mm} / \mathrm{min}$. The load was measured with a $5 \mathrm{kN}$ Xforce HP load cell.

\section{Results and discussion}

\subsection{Results}

Theoretical considerations that describe cracks as surface dipoles to interpret their emitted electromagnetic signals have been published before [29]. Assuming the applicability of the quasistatic approximation [30], dipole like EME sources, i.e. asymmetrically charged crack surfaces (with a total charge $Q=0$ ), as well as crack dimensions that are small compared to the sensor dimensions, the electric potential $\Phi$ at a position $\mathbf{r}$ (position with respect to the centre of the sensor array) can be approximated by:

$\Phi(\mathbf{r}, t)=\frac{\mathbf{p}(t) \cdot \mathbf{r}}{4 \pi \varepsilon_{0} \mathrm{r}^{3}}$

with the general definition of the dipole moment:

$\mathbf{p}(t)=\int_{V^{\prime}} \mathbf{r}^{\prime} \rho\left(\mathbf{r}^{\prime}, t\right) d^{3} r^{\prime}$

Here, $\rho\left(\mathbf{r}^{\prime}, t\right)$ is the time dependent charge density representing the charged crack surfaces, i.e the generation and relaxation of surface charges as well as the crack dynamics such as crack propagation and crack surface vibrations. $V^{\prime}$ is the volume containing the damage within the specimen. For surface dipoles the dipole moment acts perpendicular to the crack surface (i.e. parallel to the surface normal).

An inverse use of equation (1) points out a useful application, namely the determination of the dipole orientation and therefore the orientation of the crack surfaces. For the sake of simplicity, a restriction has to be made. While varying the source-sensor orientation the distance between source and sensor has to remain approximately constant. Else, the $1 / r^{2}$ dependence of the dipole potential may have a significant effect on the signal amplitude that may override the influence of the angular dependence. In cases were this cannot be assured by the experimental set-up, the distances have to be determined as well. Another huge influence on the determination of the source orientation are effects that distort the assumed dipole-like field distribution. Any conductor in the vicinity of the source changes the distribution of the field due to near-field interaction with the conductor causing induced charges on it's surface. Thus it is necessary to avoid conducting elements near the source and the sensors for this kind of measurement. Therefore, we have chosen to fabricate our load fixtures from non-conducting materials, leaving only the sensors and the shielding enclosure conductive. Bending test fixtures made from PMMA were already presented for this purpose [11]. Nevertheless, possible influences to the distribution of the emitted electromagnetic fields caused by the conductivity of the carbon fibres and the resulting anisotropic bulk conductivity of the tested CFRP specimens may have to be considered.

So if the electric field can be approximated by equation (1) the measurement of the electric potential in all three directions in space, with fixed source-sensor distances, allows for the determination of the orientation of the dipole moment by the ratios of the measured signal amplitudes.

For this purpose we conducted 3-point flexure tests on CFRP specimens with different ply stacking sequences with the aim to induce failure with a variety of crack surface orientations. These are governed by the chosen ply orientation, the layer thickness and the inter-play between compressive and tensile stresses at top and bottom of the plates. For the detection of the emitted EME signals we placed two EME sensors near the centre of the specimen. The sensors are arranged perpendicular to each other, with $25^{\circ}$ rotation relative to the plate normal, and extent for a certain length along the width direction of the specimen (see figure 1). Therefore, the source orientation is determined in the plane spanned by the two detection directions (quasi 2 dimensional). Figure 2 shows a schematic of the used sensor configuration. The angle $\beta$, i.e. the angle between the horizontal plane (x-y-plane) and the crack plane (see figure 2), can then be derived by

$$
\beta=\alpha-25^{\circ}=\arctan \left(A_{2} / A_{1}\right)-25^{\circ}
$$

where $\alpha$ is the angle between the surface dipole moment and the detection direction of EME sensor 1. $A_{1}$ and $A_{2}$ are the amplitudes of the EME signals detected by the respective EME sensors at a given time.

To induce the generation EME signals, the specimens are mechanically loaded until failure occurs. For 


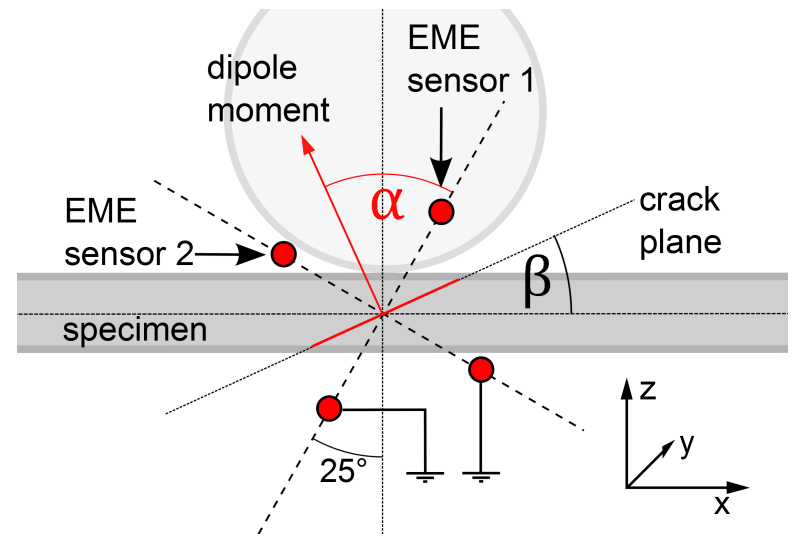

Fig. 2 Schematic for the derivation of the source orientation.

the specimens investigated here initial failure occurs in the topmost $0^{\circ}$-layer under compressive load. Multiple AE signals but only a few EME signals could be detected before the final failure of the specimen. This discrepancy in the ability to detect signals generated by microscopic failure is attributed to the different sensitivities of the AE and EME sensors. Figure 3 (top) shows an exemplary EME signal pair detected by the EME sensors during this first phase of microscopic damage. A $20 \mathrm{kHz}$ low pass filter (Butterworth, 2nd order) was applied to these weak EME signals to reduce the considerable influence of the noise floor on the subsequent calculation of the source orientation. Figure 3 (bottom) shows the angle of the EME source, i.e. the angle of the crack surface as function of time, calculated from the filtered EME signals, starting at the moment of crack initiation, using equation (3).

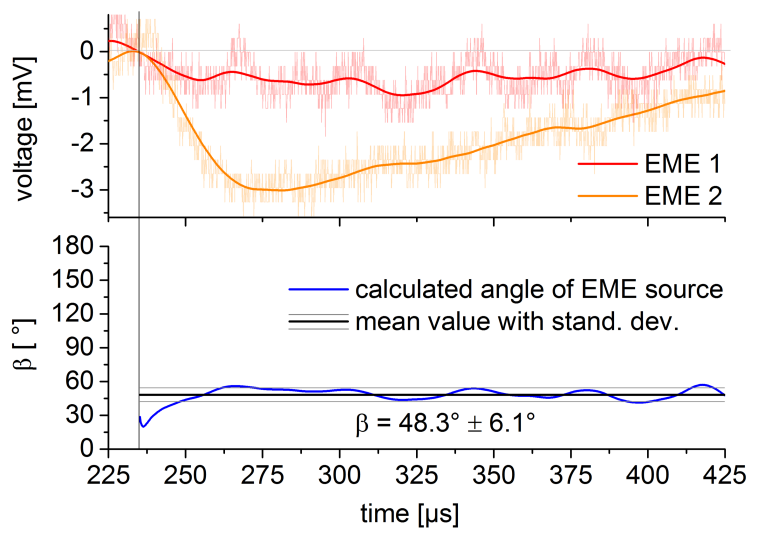

Fig. 3 Top: EME signals (raw data and low pass filtered) recorded at $95 \%$ ultimate load. Bottom: angle of the fracture surface calculated from filtered EME signals.

Ultimate failure of the specimens is accompanied by cascades of strong AE and EME signals. Figure 4 shows an exemplary EME signal pair, recorded during the fi- nal failure of a specimen and consisting of at least four different individual signals occurring in rapid succession. The recorded EME signals are plotted with two different voltage scales (figure 4 top and mid) to account for the large variation in signal strength for the individual signals. Again, the signals are low pass filtered to reduce the effect of noise. Due to smaller rise times of the individual EME signals generated in this stage a 20 $\mathrm{kHz}$ filter, as used above, would significantly alter the shape of these signals. Therefore, we here applied a 200 $\mathrm{kHz}$ Butterworth filter (2nd order) which only eliminates high frequency noise. For the exemplary EME signal, the angle of the dipole source is calculated for all times between the starting time of the EME signal and the time the first EME signal reaches saturation due to it's amplitude exceeding the operational range of the first pre-amplifier.

The variation of the calculated angles over time, shown in figures figure 3 and 4 , may contain an actual variation of the fracture surface orientation, but is mostly caused by the noise remaining after filtering. This influence is the more pronounced the weaker the EME signals are, especially at the start of the signals, and almost negligible for stronger EME signals (see figure 4 , time intervals 3 and 4 ). Therefore, for each fracture event, we assumed the fracture surface angle to be constant over time, and give its value by its temporal mean. The standard deviation then mostly only relates to the residual noise floor.

A comparison between calculated EME source orientations and actual fracture surfaces in the damaged specimen was conducted. The exemplary EME signals shown in figures 3 and 4 were recorded during the fracture test of the same CFRP specimen. A microscopic image (taken after the fracture test) of this very specimen (with a $\left[0_{2}, 90,0,90\right]_{\text {sym }}$ ply stacking sequence) is shown in figure 5 . The figure also contains the position of the load nose and the positions and orientations of the EME sensors. Failure of this specimen, and all other specimens, started in the topmost $0^{\circ}$-layer beneath the load nose. For a more extensive evaluation of the specimen damage and the determination of the fracture surface orientations within the specimen a (postmortem) computed tomography (CT) scan of the damaged specimen region was conducted. As can be seen in the CT images of the exemplary specimen, shown in figure 6 , two main failure modes occur in the $0^{\circ}$-layers. The major contribution of damage in this layer is given by a macroscopic crack (highlighted in pink in figure 6 , right) resulting from compressive stress. The angles and the position (in x-direction) of the fracture surfaces significantly vary along the width (y-direction) of the specimen. Measurable EME signals generated be- 


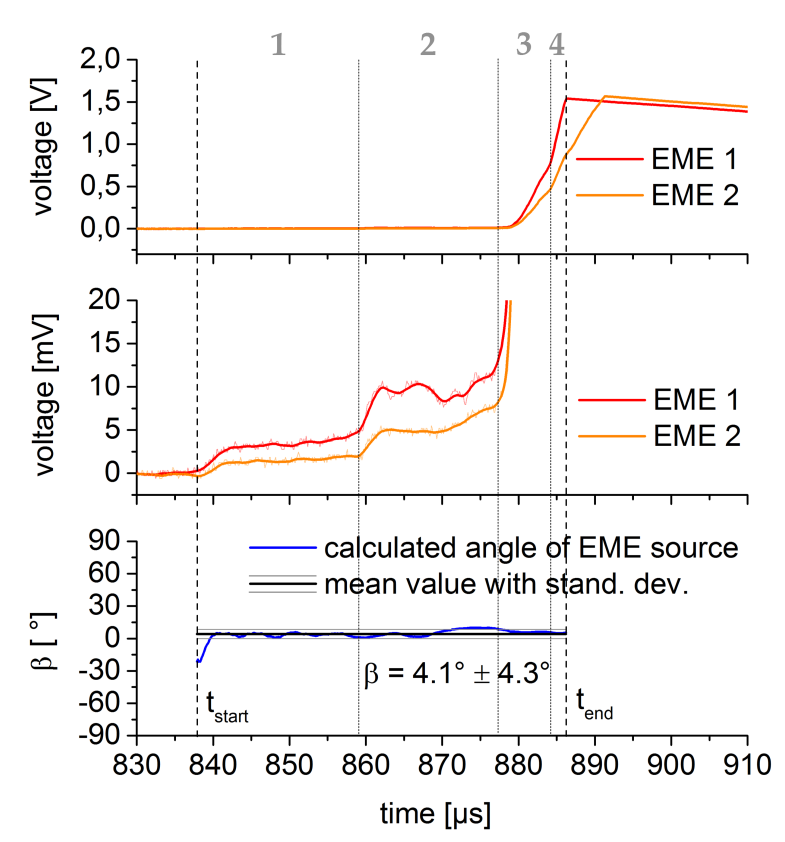

Fig. 4 Top: Sequence of four EME signals (low pass filtered) recorded during critical failure of the specimen (starting of different EME signals indicated by dotted lines). Middle: Same EME signals (raw data and low pass filtered) plotted with smaller voltage scale. Bottom: angle of the fracture surface, calculated between the time of EME signal start ( $\left.t_{\text {start }}\right)$ and time of the first EME signal amplifier saturating $\left(t_{\text {end }}\right)$.

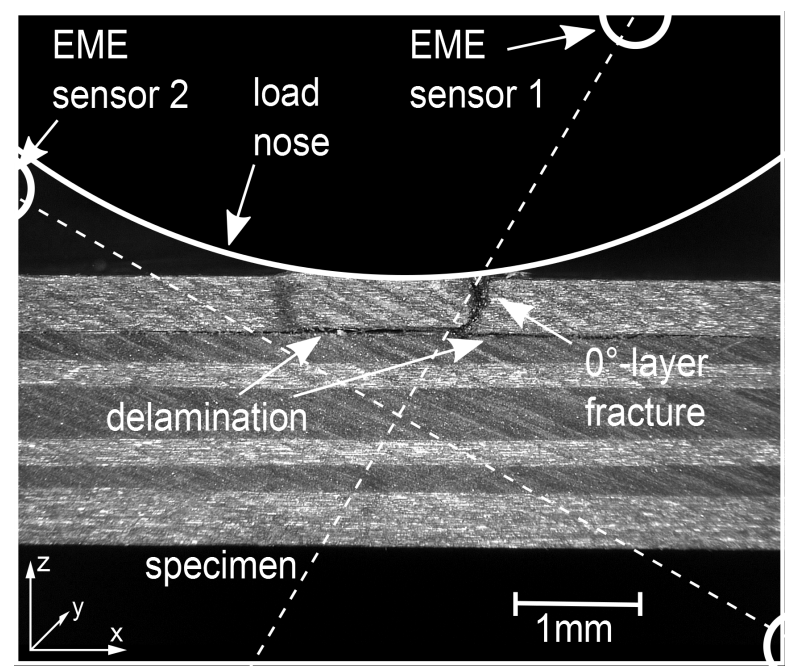

Fig. 5 Microscopic image (acquired post-mortem) of exemplary specimen $\left(\left[0_{2}, 90,0,90\right]_{\text {sym }}\right.$ ply stacking sequence) with visible damage. Positions of EME sensors and load nose are indicated.

fore ultimate specimen failure are assumed to originate from compressive fracture in this layer. The general orientation of this macroscopic crack is determined for a series of $\mathrm{x}$-z-cut planes along the width of the specimen. The crack's main angle in the topmost $0^{\circ}$ layers, derived from the $\mathrm{CT}$ scan, varies between $37^{\circ} \leq \beta_{C T, 0^{\circ}} \leq 128^{\circ}$.

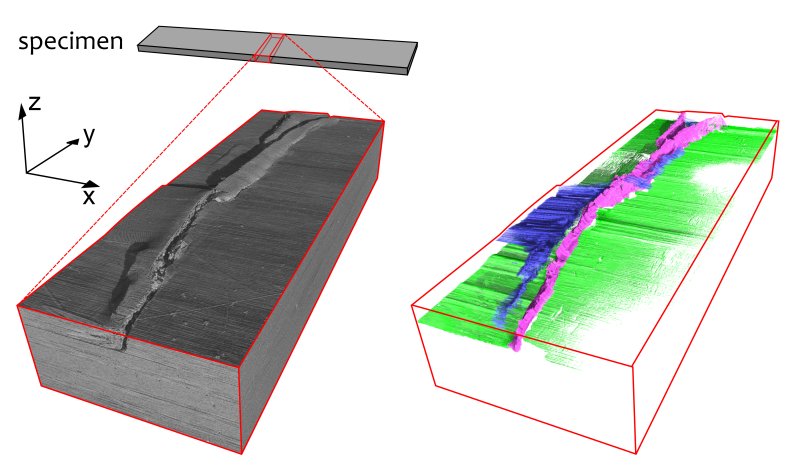

Fig. 6 Computed tomography images of exemplary specimen. Left: 3D rendering of damaged region. Right: 3D visualization of fracture surfaces: $0^{\circ}$-layer fracture (pink), intralaminar fracture (blue) and interlaminar fracture (green).

The second type of damage in the $0^{\circ}$-layers is intra-ply fracture (highlighted in blue in figure 6, right), with crack surfaces oriented mostly horizontally. The calculated angle of the source of the exemplary EME signal assumed to be emitted during the generation of fracture in this layer, is $\beta_{E M E, 0^{\circ}} \approx 48.3^{\circ} \pm 6.1^{\circ}$ (see figure $3)$.

After the fracture of the two topmost $0^{\circ}$-layers, large scale delamination between the $0^{\circ}$-layer and the adjacent $90^{\circ}$-layer occurs (see figures 5 and 6 ). This failure generates strong AE and EME signals. Even though the specimen is significantly deformed at the moment of the occurrence of this failure, the general orientation of the interlaminar crack surface plane can be approximated as $\beta_{C T, \text { interlam }} \approx 0^{\circ}$, i.e. the crack surface plane coincides with the horizontal plane ( $x-y$-plane). The calculated angle representing the orientation of the source of the exemplary EME signal (see figure 4 ) is $\beta_{E M E \text {, interlam }} \approx 3.9^{\circ} \pm 4.6^{\circ}$.

The exemplary specimen discussed so far is representative for most of the tested specimens. Regardless of the specific ply stacking sequence, the sequence of failure almost always occurred in a similar way. Fracture of the topmost $0^{\circ}$-layers with a mostly vertical macroscopic orientation is followed by large scale delamination between the $0^{\circ}$-layers and the adjacent $90^{\circ}$-layers. The average crack surface angle calculated from EME signals detected before final failure is $\bar{\beta}_{E M E, 0^{\circ}}=54^{\circ}$ $\pm 10^{\circ}$ while for the signals generated during ultimate failure the average crack plane angle was calculated to be $\bar{\beta}_{E M E \text {,interlam }}=4^{\circ} \pm 8^{\circ}$.

\subsection{Discussion}

For the EME signals emitted before ultimate failure the calculated angles are within the range of the actual crack surface angles. Nevertheless, the accuracy of 
this source orientation determination suffers from various influences. Since the weak EME signals emitted in this stage originate from smaller cracks in this layer and not necessarily the full macroscopic crack, the exact origins of the signals and therefore their orientation remain unclear. Figure 5 reveals another probable cause for the discrepancies between calculated and actual fracture surface angles. The distance between EME source and sensor cannot always be identical for the two EME sensors. Therefore, equation 3 does not yield the best approximation since it requires the source to be in the centre of the sensor array. In the present case, as mentioned in the introduction of this section, a calculation that takes into account the actual position of the source would certainly prove more adequate. Moreover, effects caused by the anisotropic electric properties of the tested CFRP specimen were not considered but certainly influence the distribution of the electric field. Furthermore, the weak EME signals generated by this early damage are superimposed by the permanent noise floor which, even after noise reduction, influences the results of the crack angles calculation.

As for the final failure of the tested specimens, the sources, i.e. the delamination planes, are also not located precisely at the centre of the EME sensors (see figure 5). Nevertheless, the presented approach seems to result in calculated orientations, which are in fairly good agreement with the measured orientation.

In particular, the resulting source orientations derived from the first EME signals can be clearly distinguished from the ones occurring during the final failure.

A first step to improve this method is to take into account the actual source position. A possible approach could be the determination of the cracks' locations by means of AE source localization procedures. However, this would require the application of the presented fracture surface angle determination method on a slightly larger scale since the accuracy of standard and even advanced $\mathrm{AE}$ source location methods is limited to some millimetres [ref paper? evtl. sinans?]. As increasing sourcesensor-distances in the presented set-up would greatly reduce the sensitivity of the EME detection, the application of more sophisticated EME sensor arrays spanning a larger area of the specimens could be considered.

\section{Conclusion}

In this manuscript we demonstrated a possible method to determine the orientation of fracture surfaces in CFRP by taking advantage of the directional character of the fracture induced electromagnetic emission.
By applying two EME sensors with perpendicular measurement directions and by assuming a basic model for the EME generating cracks as small surface dipoles (point dipoles), the orientation of the dipole moment and therefore the orientation of the EME source can be calculated from the ratio of the measured EME signal's amplitudes. Even with this simplified assumption we were able to clearly distinguish different fracture orientations in CFRP by their different orientation within the tested specimens as validated by computed tomography measurements.

Improvements of the measurement equipment, e.g. by using more than two EME sensors with different detection directions, and refinements of the model used for the calculations, e.g. applying numerical modelling, should result in an increased accuracy of this method.

Accessing the orientation of failure in complex, multilayer composite structures is a substantial advantage provided by EME measurements and promises a range of possible applications, especially when combined with other non-destructive testing methods such as acoustic emission.

Acknowledgements This research is funded by the DFG as part of the project "Relation of electromagnetic and acoustic emission to temporal and spatial crack motion on a microscopic scale in polymers and carbon fibres".

\section{References}

1. Yamada I, Masuda K, Mizutani H, Electromagnetic and acoustic emission associated with rock fracture. Phys. Earth Planet. Int. 57 157-68 (1989)

2. Rabinovitch A, Frid V, Bahat D, Goldbaum J, Fracture area calculation from electromagnetic radiation and its use in chalk failure analysis. Int. J. Rock Mech. Min. Sci. 37 1149-54 (2000)

3. Koshevaya S, Grimalsky V, Makarets N, Kotsarenko A, Siquieros-Alatorre J, Perez-Enriquez R, Juarez-Romero $\mathrm{D}$, Electromagnetic emission from magnetite plate cracking under seismic processes. Adv. Geosci. 14 25-28 (2008)

4. Lacidogna G, Carpinteri A, Manuello A, Durin G, Schiavi A, Niccolini G, Agosto A, Acoustic and Electromagnetic Emissions as Precursor Phenomena in Failure Processes. Strain 47 144-52 (2010)

5. Mori Y, Obata Y, Sikula J, Acoutic and electromagnetic emission from crack created in rock samples under deformation. J. Acoustic Emission 27 157-66 (2009)

6. Frid V, Rabinovitch A and Bahat D, Fracture induced electromagnetic radiation. J. Phys. D: Appl. Phys. 36 1620-8 (2003)

7. Rabinovitch A, Frid V, Goldbaum J, Bahat D, Polarization-depolarization process in glass during percussion drilling. Philos. Mag. 83 2929-40 (2003)

8. Aman S, Aman A, and Tomas J, Method of Crack Formation Analysis Based on Mechanoluminecence. Mater. Sci. Appl. 3 739-44 (2012) 
9. Misra A, Prasad R C, Chauhan V S, Srilakshmi B, A theoretical model for the electromagnetic radiation emission during plastic deformation and crack propagation in metallic materials. Int. J. Fract. 145 99-121 (2007)

10. Dickinson J T, Jensen L C, Dion R P, Fracto-emission from high density polyethylene: Bond breaking versus tribological stimulation. J. Appl. Phys. 73 3047-54 (1993)

11. Gade S O, Weiss U, Peter M A and Sause M G R, Relation of Electromagnetic Emission and Crack Dynamics in Epoxy Resin Materials. J. Nondestruct. Eval. 33 711-23 (2014)

12. Petrenko V F, Electromechanical phenomena in ice. CRREL Special report 96-2 (1996)

13. Mizuno, Y, Light emission associated with deformation and fracture of ice. J. Jpn. Soc. Snow 64 241-8 (2002)

14. Dickinson J T, Jahan-Latibari A, Jensen L C, Electron emission and acoustic emission from the fracture of graphite/epoxy composites. Int. Mater. Sci. 20 229-36 (1985)

15. Astanin V V, Shchegel G O, Hufenbach W, Hornig A, Langkamp A, Characterising failure in textile-reinforced thermoplastic composites by electromagnetic emission measurements under medium and high velocity impact loading. Int. J. Impact. Eng. 49 22-30 (2012)

16. Sedlak P, Enoki M, Ogasawara T, Sikula J, Electromagnetic and acoustic emission in PEEK/Carbon nanotube composites. 29th European Conference on Acoustic Emission Testing, Vienna (2010)

17. Stoudek R, Trcka T, Matysik M, Vymazal T, Plskova I, Acoustic and electromagnetic emission of lightweight concrete with polypropylene fibers. Mater. Tehnol. 50 547-52 (2016)

18. Koktavy P, Experimental study of electromagnetic emission signals generated by crack generation in composite materials. Meas. Sci. Technol. 20 8pp (2009)

19. Sklarczyk C, Winkler S, Thielicke B, Die elektrische Emission beim Versagen von Faserverbundwerkstoffen und ihren Komponenten. Mat.-wiss. u. Werkstofftech. 27 559-66 (1996)

20. Macku R, Koktavy P, Trcka T, Sicner J, Fracture Related Electromagnetic Emission Measurement and Excess Noise Analysis of Reinforced Composites. Proc. Mater. Sci. 3 116-21 (2014)

21. Sause M G R, In-situ monitoring of fiber-reinforced composites. Springer-International, Cham (2016)

22. Tsutsumi A, Shirai N, Electromagnetic signals associated with stickslip of quartz-free rocks. Tectonophysics. 450 79-84 (2008)

23. Sedlak P, Sikula J, Lokajicek T, Mori Y, Acoustic and electromagnetic emission as a tool for crack localization. Meas. Sci. Technol. 19 7pp (2008)

24. Takeuchi A, Nagahama H, Electric dipoles perpendicular to a stick-slip plane. Phys. Earth Planet In. 155 208-18 (2006)

25. DIN EN ISO 14125 Fibre-reinforced plastic composites Determination of flexural properties (1998)

26. DIN EN ISO 291 Plastics - Standard atmospheres for conditioning and testing (2008)

27. Partridge R E, Capacitive probe E-field sensors. Sensor and simulation notes 11 17pp (1965)

http://ece-research.unm.edu/summa/notes/SSN/note11. pdf

28. Baum C, Breen E, Giles J, O'Nelll J and Sower G, Sensors for Electromagnetic Pulse Measurements Both Inside and Away from Nuclear Source Regions. IEEE Trans. Electromagn. Compat. 26 22-35 (1978)
29. Ivanov V V, Egorov P V, Kolpakova L A and Pimonov A G (1988) Crack dynamics and electromagnetic emission by loaded rock masses. Sov. Min. Sci. 24 406-12

30. Dirks H K, Quasi-stationary fields for microelectronic applications. Electr. Eng. 79 145-55 (1996) 